\title{
EMPRESAS EXITOSAS Y NO EXITOSAS DEL SECTOR DE PRODUCTOS DE CONSUMO FRECUENTE QUE COTIZAN EN LA BOLSA MEXICANA DE VALORES: UNA CLASIFICACIÓN MEDIANTE ANÁLISIS DISCRIMINANTE MÚLTIPLE
}

\section{Successful and Unsuccessful Companies in the Frequent Consumer Products Sector Listed on the Mexican Stock Exchange: A Classification Using Multiple Discriminant} Analysis

\author{
Raul Mejia Ramirez ${ }^{1}$ \\ José Antonio Echenique García² \\ Eduardo Villegas Hernández ${ }^{3}$
}

\section{RESUMEN}

El objetivo de este artículo es determinar las razones financieras que discriminan las empresas con un alto y bajo desempeño financiero del sector de productos de consumo frecuente que cotizan en la Bolsa Mexicana de Valores. Para ello, se recopilaron los estados financieros de 16 empresas desde 2001 hasta 2017, con periodicidad trimestral, con los que se calcularon variables que contemplan razones financieras de liquidez, endeudamiento, apalancamiento, RION, INVESTRAT. Para la parte metodológica primero se propone una clasificación de forma objetiva basada en el análisis de

\footnotetext{
${ }^{1}$ Doctor en Ciencias de la Administración con Mención Honorífica por la FCA-UNAM; Profesor del Tecnológico Nacional de México-ITS Costa Chica, y Profesor Visitante de la UAM-Iztapalapa, Coordinación de Administración, Área Planeación Estratégica de las Empresas. Correo electrónico: raul_mejia81@yahoo.com.mx

${ }^{2}$ Doctor en Investigación de Operaciones por la UNAM; Profesor-Investigador de la FCA-UNAM, Exdirector de dicha Facultad y actualmente presidente de su Sociedad de Egresados. Correo electrónico: jaeg21@prodigy.net.mx

${ }^{3}$ Doctor en Administración (Organizaciones); Profesor-Investigador en el Departamento de Economía de la UAM-Iztapalapa, Coordinación de Administración, Área Planeación Estratégica de las Empresas, y de la FCA-UNAM. Correo electrónico: evillegash@hotmail.com
}

Fecha de recepción: 08/02/2020 • Fecha de aceptación: 26/06/2020 
clúster para después mediante el análisis discriminante concluir que son 10 razones financieras de un total de 37 contempladas en este estudio las que más contribuyen a la discriminación; estas son: Circulante, Prueba de ácido, Apalancamiento, Veces que se paga la pérdida cambiaria, Efectivo de operación interés, Efectivo de operación a pérdida cambiaria, Días de cobro, Rotación del activo, Margen de utilidad neta y Rentabilidad del capital contable.

Palabras clave: Desempeño financiero, análisis clúster, análisis discriminante, razones financieras.

Clasificación ]EL: C59, G11.

\section{ABSTRACT}

The objective of this article is to determine the financial reasons that discriminate the companies, with a high and low financial performance, of the sector of frequent consumption products that are quoted in the Mexican Stock Market. For this, the financial statements of 16 companies were compiled from 2001 to 2017 on a quarterly basis, with which variables were calculated that include financial ratios of liquidity, indebtedness, leverage, RION, INVESTRAT. For the methodological part, an objective classification based on the cluster analysis is first proposed, and then through the discriminant analysis, concluding that there are 10 financial reasons out of 37 considered in this study, which more contribute to discrimination, being: Current money, Acid test, Leverage, Times that the exchange loss is paid, Operating cash interest, Cash operating at foreign exchange loss, Collection days, Rotation of assets, Net profit margin and Return on equity.

Keywords: Financial performance, cluster analysis, discriminant analysis, financial reasons.

JEL Classification: C59, G11.

\section{Introducción}

os estados financieros permiten evaluar los resultados operativos y condiciones financieras de la empresa y sirven como insumo principal en los diversos modelos existentes para discriminar entre empresas con alto y bajo desempeño financiero. 
El análisis de clúster en los estudios sectoriales permite clasificar a las empresas en grupos homogéneos con la finalidad de facilitar la consecución de los objetivos de la investigación ya que permite llegar a resultados más particulares de las características del sector en estudio.

El análisis discriminante permite realizar una evaluación detallada del desempeño financiero de las empresas, tomando como base las razones financieras y definiendo las variables dependientes e independientes con la finalidad de disminuir la varianza dentro de los grupos y maximizar la varianza entre grupos.

El sector de productos de consumo frecuente no ha sido estudiado desde el punto de vista del colectivo de empresas que cotizan en la Bolsa Mexicana de Valores (BMV) empleando el análisis de clúster y el análisis discriminante, lo cual justifica esta investigación.

Debido a lo anterior, el objetivo de este trabajo es determinar las razones financieras que permiten discriminar a las empresas de sector de productos de consumo frecuente que cotizan en la BMV, en cuanto a su nivel de desempeño financiero, alto o bajo, mediante la aplicación del análisis discriminante, en donde las razones financieras de liquidez, actividad, rentabilidad y endeudamiento actúan como variables independientes y los niveles de desempeño financiero, alto y bajo, como variables dependientes. Esto último, con el objeto de analizar la relación existente entre en nivel de desempeño financiero de las empresas y la incidencia de las razones financieras seleccionadas.

Para la realización de este trabajo, se recopiló la información financiera oficial que presentan las empresas en forma trimestral a la BMV. Dicha información va desde el cuarto trimestre del año 2001 hasta el tercer trimestre de 2015. Se contemplaron 19 empresas mexicanas del sector en estudio, las cuales son: AC, BACHOCO, BAFAR, BIMBO, CHDRAUI, COMERCI, CULTIBA, FEMSA, GICANTE, GRUMA, HERDEZ, INGEAL, KIMBER, KOF, LALA, MASECA, MINSA, SORIANA, WALMEX.

\section{Fundamentos teóricos y revisión de la literatura}

El uso de las razones financieras surge cuando los inversionistas de Estados Unidos comenzaron a utilizarlas como técnica de gestión y administración, basándose en el análisis financiero cuyo pionero fue Alexander Wall, después del crack bursátil de 1929, periodo que se caracterizó por quiebras masivas de empresas estadounidenses (Ibarra, 2010). 
Con lo anterior surgen los primeros trabajos y modelos para predecir la insolvencia, basados en razones financieras, los cuales pueden ser divididos en tres etapas. La primera etapa consistía en llevar a cabo el análisis financiero a través de razones financieras y conocer el valor que dichas razones aportaban como herramienta de predicción (Ibarra, 2010).

En esta etapa surge el trabajo pionero de (Fizpatrick, 1931), donde tomó una muestra de 19 empresas sanas y 19 empresas en quiebra determinando que los factores de predicción más importantes eran la rentabilidad y el endeudamiento. Es importante mencionar que dichos resultados carecían de exactitud debido a la falta de tecnología, y a la forma de registro y presentación de los estados financieros (Ibarra, 2010).

La segunda etapa se constituye básicamente con la creación de los primeros modelos, los cuales consistían en modelos univariables que tenían como objetivo principal la utilización por separado de las variables independientes con el fin de explicar una variable dependiente (Ibarra, 2010).

Beaver (1966) representa dicha etapa mediante sus estudios empíricos a través de la utilización de las razones financieras con el fin de poder determinar la solvencia y liquidez de las empresas para posteriormente predecir una quiebra. Sin embargo, más que determinar la mejor herramienta de predicción, William Beaver quería señalar la importancia de la información contable y su utilidad de predicción, ya que en el pasado lo anterior no había sido empíricamente contrastado (Ibarra, 2010).

La tercera etapa es aquella donde aparecen los modelos multivariantes los cuales analizan simultáneamente tres o más variables independientes. Dentro de esta etapa el principal estudio realizado fue el modelo de Z-Score de (Altman, Financial ratios, discriminant analysis, and the prediction of corporate bankruptcy, 1968) mediante el método de análisis discriminante debido a la capacidad significativa para predecir la insolvencia (Ibarra, 2010).

Edward Altman en su modelo Z-Score, definía insolvencia como la falta de pago a los bancos por parte de las empresas. Su primer modelo utilizó una muestra de 66 empresas de la industria manufacturera, 33 empresas que habían sido insolventes y 33 financieramente solventes, en un periodo de 1946 a 1965 (Somoza, 2000).

Utilizó una combinación de 5 razones financieras que fueron: capital de trabajo a activo total. Utilidades retenidas a activo total, utilidades antes de intereses e impuestos a activo total, valor de mercado del capital contable a valor en libros 
de la deuda y ventas a activo total. Usando esas razones en un modelo, obtenía un valor Z, el cuál determinaba el rango donde se clasificaba la empresa. Si el valor $Z$ era menor o igual a 2.675 la empresa caía como insolvente y si era mayor estaba en una zona segura. Altman concluyó su investigación demostrando que su modelo tuvo un porcentaje de precisión del 95\% para el primer año (Somoza, 2000).

Para 1977, Altman determinó la necesidad de realizar un nuevo estudio, por lo que junto con Haldeman y Narayanan, realizaron una nueva versión del modelo de 1968 al denominaron ZETA. En este modelo se utilizó una muestra de 111 empresas, 53 en bancarrota y 50 sanas en un periodo de 1969 a 1975, lo que diferenciaba con el de 1968, es que en el ZETA, se incluían empresas de venta al detalle además de las manufactureras. El objetivo de esta nueva versión era poder incrementar la predicción de la insolvencia para los años posteriores (Altman, Hadelman, \& Narayanan, 1977).

Los cambios que realizaron fueron: en la muestra cambiaron el tamaño e incluyeron a empresas de venta al detalle, en cuanto a las variables analizaron las notas de los estados financieros para calcular las razones financieras y utilizaron información más reciente (Altman, Hadelman, \& Narayanan, 1977).

Las conclusiones de la investigación resultaron exitosas ya que las modificaciones lograron que para el año 5 se predijera la insolvencia con una precisión del $70 \%$ mientras que en el estudio de 1968, esa precisión se lograba para el año 2 (Altman, Hadelman, \& Narayanan, 1977).

Una de las investigaciones recientes basada en el modelo Z-Score de Altman, fue realizada en el 2009 por los profesores de la Universidad de Kentucky de EUA, el objetivo principal era eliminar las críticas al estudio anterior, utilizando una muestra más grande que incluyera empresas de diversas industrias, empleando datos recientes y de diversos años (Russ, Achilles, \& Greenfield, 2009).

\section{Marco referencial}

La evaluación de empresas supone un cuerpo de conocimientos teóricos y metodológicos, así como un conjunto de habilidades aplicadas. Para este trabajo este cuerpo de conocimientos lo constituyen los fundamentos del Análisis Discriminante (AD), que permite discriminar las razones financieras como liquidez, endeudamiento, eficiencia, rentabilidad, crecimiento, RION, GEO e INVESTRAT que determinan el 
nivel de desempeño financiero de las empresas del sector de productos de consumo frecuente que cotizan en la BMV.

\subsection{Indicadores financieros}

Los indicadores financieros son el producto de establecer resultados numéricos basados en relacionar dos cifras o cuentas bien sea del Balance Ceneral y/o del Estado de Pérdidas y Ganancias. Los resultados así obtenidos por sí solos no tienen mayor significado; sólo cuando son relacionados unos con otros y son comparados con los de la operación de la compañía, se pueden obtener resultados significativos y sacar conclusiones sobre la situación financiera real de la empresa, siendo el objeto de estudio de esta investigación las empresas del sector de productos de consumo frecuente que cotiza en la BMV (Vázquez, Guerra, \& Ahmed, 2008).

En concordancia con lo anterior y teniendo en cuenta que los indicadores antes mencionados permiten clasificar a las empresas en estudio, en grupos homogéneos, que en esencia es el propósito de la evaluación del mejoramiento de los indicadores seleccionados, en este trabajo se identificaron y calcularon los siguientes indicadores financieros:

\section{Indicadores de liquidez}

Estos indicadores surgen de la necesidad de medir la capacidad que tienen las empresas para cancelar sus obligaciones a corto plazo. Sirven para establecer la facilidad o dificultad que presenta una compañía para pagar sus pasivos corrientes con el producto de convertir a efectivo sus activos corrientes. Determinan que pasaría si la empresa se le exigiera el inmediato de todas sus obligaciones a menos de un año. En el Cuadro 1 se presentan las ecuaciones (1), (2) y (3) que indican cómo calcular los indicadores de liquidez (Circulante, Prueba de ácido y Prueba rápida).

CUADRO 1. INDICADORES DE LIQUIDEZ

\begin{tabular}{ll}
\hline Indicador & \multicolumn{1}{c}{ Ecuación } \\
\hline Circulante & $\begin{array}{l}\text { Activo Circulante } \\
\text { Pasivo Circulante }\end{array}$ \\
\hline Prueba de ácido & Activo Circulante-Almacén \\
\cline { 2 - 3 } Prueba rápida & Pasivo Circulante \\
\hline & Efectivo y Valore \\
\hline
\end{tabular}




\section{Indicadores de endeudamiento}

Los indicadores de endeudamiento tienen por objeto medir en qué grado y de qué forma participan los acreedores dentro del financiamiento de la empresa. De la misma manera, se trata de establecer el riesgo que corren tales acreedores, el riesgo de los dueños y la conveniencia o inconveniencia de un determinado nivel de endeudamiento para la empresa, como se muestra en el Cuadro 2, ecuaciones (4), (5), (6), (7) y (8).

CuAdRo 2. INDICADORES DE ENDEUDAMIENTO

\begin{tabular}{lc}
\hline Indicador & \multicolumn{1}{c}{ Ecuación } \\
\hline Endeudamiento & $\frac{\text { Pasivo Total }}{\text { Activo Total }}$ \\
\hline \multirow{2}{*}{ Apalancamiento } & Pasivo de Largo Plazo \\
\cline { 2 - 2 } $\begin{array}{l}\text { Veces que se paga el } \\
\text { interés }\end{array}$ & Activo de Largo Plazo+Capital Contable \\
\hline $\begin{array}{l}\text { Veces que se paga la } \\
\text { pérdida cambiaria }\end{array}$ & Utilidad en Operación \\
\hline $\begin{array}{l}\text { Grado de apalanca- } \\
\text { miento financiero }\end{array}$ & Utilidad en Operación \\
\hline & Pérdida Cambiaria \\
\hline
\end{tabular}

CUADRO 3. INDICADORES DE PRESIÓN FINANCIERA

\begin{tabular}{lc}
\hline Indicador & Ecuación \\
\hline \multirow{2}{*}{ Interés en ventas } & $\frac{\text { Interés Pagado }}{\text { Ventas }}$ \\
\hline \multirow{2}{*}{ Interés a utilidad neta } & $\frac{\text { Interés Pagado }}{U \text { Utilidad del Ejercicio }}$ \\
\hline $\begin{array}{l}\text { Efectivo de operación } \\
\text { interés }\end{array}$ & Recursos Generados por la Operación \\
\cline { 2 - 2 } & Interés Pagado \\
\hline Efectivo de operación a & Recursos Generados por la Operación \\
\cline { 2 - 2 } pérdida cambiaria & Pérdida Cambiaria \\
\hline
\end{tabular}




\section{CUADRO 4. INDICADORES DE EFICIENCIA}

\begin{tabular}{ll}
\hline Indicador & \multicolumn{1}{c}{ Ecuación } \\
\hline Días de pago & $\frac{\text { Cuentas por Pagar }}{\text { Compras }} \times 360$ \\
\hline Rotación de inventarios & $\frac{\text { Costo de Ventas }}{\text { (Inventario Inicial+Almacén)/2 }}$ \\
\hline Días de inventario & $\frac{360}{\text { Rotación de Inventarios }}$ \\
\hline Días de cobro & $\frac{\text { Cuentas por Cobrar }}{\text { Ventas }} \times 360$ \\
\hline Rotación del activo & $\frac{\text { Ventas }}{\text { Activo Total }}$ \\
\hline Rotación del activo de largo plazo & $\frac{\text { Ventas }}{\text { Activo de Largo Plazo }}$ \\
\hline Margen de utilidad bruta & Utilidad Bruta \\
\cline { 2 - 2 } Gastos de operación & Ventas \\
\hline Margen de utilidad neta & $\frac{\text { Gastos de Operación }}{\text { Ventas }} \times 100$ \\
\hline
\end{tabular}

\section{Indicadores de rentabilidad}

Los indicadores de rentabilidad, denominados también de rendimiento o lucratividad, sirven para medir la efectividad de la administración de la empresa para controlar el costo y el gasto, y de esta manera convertir las ventas en utilidades; como se muestra en el Cuadro 5, ecuaciones (22) y (23).

\section{CUADRO 5. INDICADORES DE RENTABILIDAD}

\begin{tabular}{ll}
\hline Indicador & Ecuación \\
\hline Rentabilidad del activo & $\frac{\text { Utilidad del Ejercicio }}{\text { Activo Total }}$ \\
\hline Rentabilidad del capital contable & $\frac{\text { Utilidad del Ejercicio }}{\text { Capital Contable }} \times 100$ \\
\hline
\end{tabular}


CUADRO 6. INDICADORES DE CRECIMIENTO

\begin{tabular}{lll}
\hline Indicador & Ecuación \\
\hline Ventas & & (24) \\
\hline Utilidad en operación & $\left(\begin{array}{c}\text { Utilidad en Operación del Periodo Actual } \\
\text { Utilidad en Operación del Periodo Anterior }\end{array}-1\right) \times 100$ \\
\hline Utilidad del ejercicio & $\left(\frac{\text { Utilidad del Ejercicio del Periodo Actual }}{\text { Utilidad del Ejercicio del Periodo Anterior }}-1\right) \times 100$ \\
\hline Generación de efectivo & $\left(\frac{\text { Recurso Generados por la Operación del Periodo Actual }}{\text { Recurso Generados por la Operación del Periodo Anterior }}-1\right) \times 100$ \\
\hline Activo & $(27)$ \\
\hline Activo de Largo Plazo & $\left(\frac{\text { Activo de Largo Plazo del Periodo Actual }}{\text { Activo de Largo Plazo del Periodo Anterior }}-1\right) \times 100$ \\
\hline Pasivo & $\left(\frac{\text { Pasivo Total del Periodo Actual }}{\text { Pasivo Total del Periodo Anterior }}-1\right) \times 100$ \\
\hline Pasivo de largo plazo & $\left(\frac{\text { Pasivo de Largo Plazo del Periodo Actual }}{\text { Pasivo de Largo Plazo del Periodo Anterior }}-1\right) \times 100$ \\
\hline Capital contable & $\left(\frac{\text { Capital Contable del Periodo Actual }}{\text { Capital Contable del Periodo Actual }}-1\right) \times 100$ & (30) \\
\hline
\end{tabular}

\section{Indicadores RION y GEO}

El indicador Ceneración Económica Operativa (CEO) es una herramienta que permite calcular y evaluar la riqueza generada por la empresa teniendo el nivel de riesgo con que opera, el indicador GEO es el importe que queda una vez que se han deducido de los ingresos la totalidad de los gastos de operación incluido el costo de oportunidad del capital y los impuestos.

\section{CuAdro 7. RION y GEO}

\begin{tabular}{lc}
\hline Indicador & \multicolumn{1}{c}{ Ecuación } \\
\hline Rendimiento de la IONP(RION) & Utilidad en Operación \\
\cline { 2 - 2 } Tasa de provisiones & Inversión Operativa Neta Promedio (IONP) \\
\cline { 2 - 2 } & Cosultado Antes de Impuestos \\
RION después de impuestos & $\times\left(-1 \frac{\text { Tasa de Provisiones }}{100}\right)$ \\
\hline
\end{tabular}


CUADRO 8. INVESTRAT

\begin{tabular}{ll}
\hline Indicador & \multicolumn{1}{c}{ Ecuación } \\
\hline \multirow{2}{*}{ Capital de deuda \% } & Capital de Deuda \\
\cline { 2 - 2 } & Inversión Estratégica \\
\hline & Capital de Aportación \\
\cline { 2 - 2 } & Inversión Estratégica \\
\hline
\end{tabular}

\section{Metodología}

Puesto que el objetivo del estudio es analizar las razones financieras que influyen en el desempeño financiero de las empresas del sector de productos de consumo frecuente que cotizan en la Bolsa Mexicana de Valores, se ha procedido a plantear un análisis en dos etapas sobre la base de datos:

Primeramente se plantea un análisis clúster tomando como variables clasificadoras las razones financieras mencionadas en el apartado 3.1, obteniendo dos grupos: 1) Alto desempeño financiero y 2) Bajo desempeño financiero.

Posteriormente, se aplica un análisis discriminante obteniendo una función discriminante que permite clasificar las empresas en alguno de los dos grupos obtenidos en primera fase.

\section{Población}

La población de estudio está conformada por 19 empresas pertenecientes al sector de productos de consumo frecuente que cotizan en la BMV que presentaron sus estados financieros a la BMV a partir del cuarto trimestre del año 2001 hasta el tercer trimestre del año 2015, las cuales son: AC, BACHOCO, BAFAR, BIMBO, CHEDRAUI, COMERCI, CULTIBA, FEMSA, GICANTE, GRUMA, HERDEZ, INGEAL, KIMBER, KOF, LALA, MASECA, MINSA, SORIANA, WALMEX.

\section{Fuentes y datos}

De la misma forma, se toman como fuentes los estados financieros que presentan las empresas de forma trimestral a la BMV. Dicha información abarca del cuarto trimestre del año 2001 hasta el tercer trimestre del año 2015. Una vez obtenidos los estados financieros se obtuvieron las distintas razones financieras mencionadas 
en el apartado 1 y se procede a realizar el análisis de la información y obtención de los modelos empleando la técnica de análisis discriminante.

\section{Análisis}

Para analizar las variables asociadas a las razones financieras seleccionadas se utilizó el análisis clúster y análisis discriminante por medio de software SPSS statistics 21.0, aplicación con la cual se obtiene el modelo propuesto; estas técnicas permiten la estimación en un marco único para analizar si las razones financieras evaluadas presentan diferencias significativas en los niveles de desempeño financiero, alto y bajo.

\section{Resultados}

Para establecer una diferenciación de las 19 empresas se podía haber acudido a los criterios clásicos de clasificación realizados de forma subjetiva por otros autores, los cuales se basan en solo unas razones financieras; sin embargo, en esta investigación se aplica una clasificación objetiva basada en el análisis clúster, el cual, a diferencia del método anterior, toma en cuenta todas las razones financieras. Debido a que la información proporcionada se refiere a datos métricos en escala de intervalo se utilizó la medida de la distancia euclidiana al cuadrado. Por otro lado, como las razones financieras se encuentran en diferentes escalas y unidades de medición se procede a la estandarización de las mismas antes de proceder con el análisis clúster. El procedimiento de aglomeración es jerárquico empleando el método del vecino más próximo, debido a que el número de empresas es relativamente pequeño. De acuerdo al análisis clúster las empresas pueden clasificarse de acuerdo a su desempeño financiero en dos grupos, los cuales se describen a continuación:

Conglomerado 1. Empresas con alto desempeño financiero. Formado por 13 empresas las cuales son: LALA, MASECA, BACHOCO Y KIMBER. Alta liquidez y alta rentabilidad.

Conglomerado 2. Empresas con bajo desempeño financiero. Formado por 6 empresas, las cuales son: AC. BAFAR, BIMBO, CHEDRAUI, COMERCI, CULTIBA, FEMSA, GIGANTE, GRUMA, HERDEZ, INGEAL, KOF, MINSA, SORIANA Y WALMEX. Se caracterizan por tener baja liquidez y baja rentabilidad. 
Una vez obtenidos los dos conglomerados en la fase anterior, se realiza un análisis discriminante que permita predecir, a partir del resto de razones financieras, la pertenencia de cada observación anual a uno u otro grupo.

A continuación se muestran los resultados del análisis discriminante (estadisticos por pasos):

Los contrastes de igualdad de medias entres los dos grupos para cada razón financiera se muestran en el Cuadro 9. Las ANOVAS indican que no se observan diferencias significativas entre los niveles de desempeño financiero, alto y bajo, en cuanto grado de apalancamiento financiero, efectivo de operación a pérdida cambiaria, margen de utilidad bruta, generación de efectivo, activo, activo de largo plazo, pasivo de largo plazo, capital contable y tasa de provisiones. En consecuencia, las razones financieras anteriores no deberían tener gran influencia a la hora de clasificar a las empresas en uno u otro grupo. En el mismo cuadro, se observa que en estos casos, el p-valor> 0.05, lo que significa que se rechaza la hipótesis nula, es decir, los grupos en media son iguales.

Las variables son introducidas/eliminadas del modelo en la medida en que tengan asociado un menor valor del estadístico $\wedge$ de Wilks.

\section{CuAdro 9. PRUEBA DE ICUALDAD DE LAS MEDIAS DE LOS GRUPOS}

\begin{tabular}{llllll}
\hline & $\begin{array}{c}\text { Lambda de } \\
\text { Wilks }\end{array}$ & \multicolumn{1}{c}{$\mathbf{F}$} & gl1 & gl2 & Sig. \\
\hline Circulante & 0.342 & 1896.975 & 1 & 987 & 0.000 \\
\hline Prueba de ácido & 0.429 & 1315.377 & 1 & 987 & 0.000 \\
\hline Prueba rápida & 0.798 & 249.368 & 1 & 987 & 0.000 \\
\hline Endeudamiento total & 0.834 & 196.834 & 1 & 987 & 0.000 \\
\hline Apalancamiento & 0.993 & 7.017 & 1 & 987 & 0.008 \\
\hline Veces que se paga interes & 0.968 & 32.835 & 1 & 987 & 0.000 \\
\hline Veces que se paga la pérdida cambiaria & 0.989 & 10.763 & 1 & 987 & 0.001 \\
\hline Grado de apalancamiento financiero & 0.997 & 3.175 & 1 & 987 & 0.075 \\
\hline Interés a ventas & 0.981 & 18.963 & 1 & 987 & 0.000 \\
\hline Interes a utilidad neta & 0.992 & 7.985 & 1 & 987 & 0.005 \\
\hline Efectivo de operación interés & 0.989 & 11.451 & 1 & 987 & 0.001 \\
\hline Efectivo de operación a pérdida cambiaria & 0.998 & 1.759 & 1 & 987 & 0.185 \\
\hline Dias de pago & 0.864 & 155.974 & 1 & 987 & 0.000 \\
\hline Rotación de inventarios & 0.853 & 170.451 & 1 & 987 & 0.000 \\
\hline Días de inventarios & 0.985 & 15.251 & 1 & 987 & 0.000 \\
\hline Días de cobro & 0.900 & 108.617 & 1 & 987 & 0.000 \\
\hline Rotación del activo & 0.978 & 22.228 & 1 & 987 & 0.000 \\
\hline Rotación del activo de largo plazo & 0.973 & 27.446 & 1 & 987 & 0.000 \\
\hline
\end{tabular}


CuAdro 9. Prueba de icualdad de las MEdias de los GRupos (CONTINUACIÓN)

\begin{tabular}{llllll}
\hline & $\begin{array}{c}\text { Lambda de } \\
\text { Wilks }\end{array}$ & F & gl1 & gl2 & Sig. \\
\hline Margen de utilidad bruta & 0.997 & 3.349 & 1 & 987 & 0.068 \\
\hline Gastosde operación & 0.962 & 39.261 & 1 & 987 & 0.000 \\
\hline Margen de utilidad neta & 0.925 & 80.463 & 1 & 987 & 0.000 \\
\hline Rentabilidad del activo & 0.957 & 44.598 & 1 & 987 & 0.000 \\
\hline Rentabilidad del capital contable & 0.984 & 16.006 & 1 & 987 & 0.000 \\
\hline Ventas & 0.995 & 4.765 & 1 & 987 & 0.029 \\
\hline Utilidad en operación & 0.983 & 17.399 & 1 & 987 & 0.000 \\
\hline Utilidad del ejercicio & 0.995 & 5.371 & 1 & 987 & 0.021 \\
\hline Generación de efectivo & 0.999 & 1.166 & 1 & 987 & 0.280 \\
\hline Activo & 0.999 & 0.960 & 1 & 987 & 0.327 \\
\hline Activo de largo plazo & 1.000 & 0.086 & 1 & 987 & 0.770 \\
\hline Pasivo & 0.993 & 6.835 & 1 & 987 & 0.009 \\
\hline Pasivo de largo plazo & 1.000 & 0.062 & 1 & 987 & 0.804 \\
\hline Capital contable & 0.999 & 1.086 & 1 & 987 & 0.298 \\
\hline Rendimiento de la ONPRION & 0.975 & 25.375 & 1 & 987 & 0.000 \\
\hline Tasa de provisiones & 0.996 & 3.640 & 1 & 987 & 0.057 \\
\hline RION después de impuestos & 0.975 & 24.977 & 1 & 987 & 0.000 \\
\hline Capital de deuda & 0.802 & 243.624 & 1 & 987 & 0.000 \\
\hline Capital de aportación & 0.801 & 245.629 & 1 & 987 & 0.000 \\
\hline
\end{tabular}

\section{Estadísticos por pasos:}

\section{CUADRO 10. VARIABLES INTRODUCIDAS/EXCLUIDAS ${ }^{A, B, C, D}$}

\begin{tabular}{|c|c|c|c|c|c|c|c|c|c|}
\hline \multirow[b]{3}{*}{ Paso } & \multirow[b]{3}{*}{ Introducidas } & \multicolumn{8}{|c|}{ Lambda de Wilks } \\
\hline & & \multirow[b]{2}{*}{ Estadístico } & \multirow[b]{2}{*}{ gl1 } & \multirow[b]{2}{*}{ gl2 } & \multirow[b]{2}{*}{ gl3 } & \multicolumn{4}{|c|}{ F exacta } \\
\hline & & & & & & Estadístico & gl1 & gl2 & Sig. \\
\hline 1 & Circulante & ,342 & 1 & 1 & 987,000 & 1896,975 & 1 & 987,000 & ,000 \\
\hline 2 & $\begin{array}{l}\text { Efectivo de } \\
\text { operación } \\
\text { a pérdida cam- } \\
\text { biaria }\end{array}$ & ,309 & 2 & 1 & 987,000 & 1100,357 & 2 & 986,000 & ,000 \\
\hline 3 & $\begin{array}{l}\text { Efectivo de ope- } \\
\text { ración interés }\end{array}$ & ,300 & 3 & 1 & 987,000 & 767,094 & 3 & 985,000 & ,000 \\
\hline 4 & Días de cobro & ,295 & 4 & 1 & 987,000 & 587.884 & 4 & 984,000 & ,000 \\
\hline 5 & Prueba de ácido & ,289 & 5 & 1 & 987,000 & 484.387 & 5 & 983,000 & ,000 \\
\hline 6 & $\begin{array}{l}\text { Rentabilidad del } \\
\text { capital contable }\end{array}$ & ,286 & 6 & 1 & 987,000 & 408.141 & 6 & 982,000 & ,000 \\
\hline
\end{tabular}


CUADRO 10. VARIABLES INTRODUCIDAS/EXCLUIDAS ${ }^{A, B, C, D}$ (CONTINUACIÓN)

\begin{tabular}{|c|c|c|c|c|c|c|c|c|c|}
\hline \multirow[b]{3}{*}{ Paso } & \multirow[b]{3}{*}{ Introducidas } & \multicolumn{8}{|c|}{ Lambda de Wilks } \\
\hline & & \multirow[b]{2}{*}{ Estadístico } & \multirow[b]{2}{*}{ gl1 } & \multirow[b]{2}{*}{ gl2 } & \multirow[b]{2}{*}{ gl3 } & \multicolumn{4}{|c|}{ F exacta } \\
\hline & & & & & & Estadístico & gl1 & gl2 & Sig. \\
\hline 7 & $\begin{array}{l}\text { Veces que se } \\
\text { paga la pérdida } \\
\text { cambiaria }\end{array}$ & ,284 & 7 & 1 & 987,000 & 353.099 & 7 & 981,000 & ,000 \\
\hline 8 & Apalancamiento & ,282 & 8 & 1 & 987,000 & 312.382 & 8 & 980,000 & ,000 \\
\hline 9 & $\begin{array}{l}\text { Margen de utili- } \\
\text { dad neta }\end{array}$ & ,279 & 9 & 1 & 987,000 & 280.653 & 9 & 979,000 & ,000 \\
\hline 10 & $\begin{array}{l}\text { Rotación del } \\
\text { activo }\end{array}$ & ,274 & 10 & 1 & 987,000 & 258.794 & 10 & 978,000 & ,000 \\
\hline
\end{tabular}

En cada paso se introduce la variable que minimiza la lambda de Wilks global.
A. El número máximo de pasos es 74
B. La F parcial mínima para entrar es 3.84
C. La F parcial máxima para salir es 2.71
D. El nivel de F, la tolerancia o el VIN son insuficientes para continuar los cálculos

CuAdRo 11. LAMBDA DE WILKS

\begin{tabular}{cccccccccc}
\hline & \multirow{2}{*}{$\begin{array}{l}\text { Núm. } \\
\text { Paso variables }\end{array}$} & Estadístico & gl1 & gl2 & gl3 & Estadístico & gl1 & gl2 & Sig. \\
\hline 1 & 1 &, 342 & 1 & 1 & 987 & 1896,975 & 1 & 987,000 &, 000 \\
\hline 2 & 2 &, 309 & 2 & 1 & 987 & 1100,357 & 2 & 986,000 &, 000 \\
\hline 3 & 3 &, 300 & 3 & 1 & 987 & 767,094 & 3 & 985,000 &, 000 \\
\hline 4 & 4 &, 295 & 4 & 1 & 987 & 587.884 & 4 & 984,000 &, 000 \\
\hline 5 & 5 &, 289 & 5 & 1 & 987 & 484.387 & 5 & 983,000 &, 000 \\
\hline 6 & 6 &, 286 & 6 & 1 & 987 & 408.141 & 6 & 982,000 &, 000 \\
\hline 7 & 7 &, 284 & 7 & 1 & 987 & 353.099 & 7 & 981,000 &, 000 \\
\hline 8 & 8 &, 282 & 8 & 1 & 987 & 312.382 & 8 & 980,000 &, 000 \\
\hline 9 & 9 &, 279 & 9 & 1 & 987 & 280.653 & 9 & 979,000 &, 000 \\
\hline 10 & 10 &, 274 & 10 & 1 & 987 & 258.794 & 10 & 978,000 &, 000 \\
\hline
\end{tabular}

\section{Resumen de las funciones canónicas discriminantes}

El Cuadro 12, aplica el contraste de significación para un eje discriminante. El estadístico del contraste de significación global Lambda de Wilks es de 0.274 
que conduce a rechazar la hipótesis nula de igualdad de medias ( $p$-valor $=0.000$ $<0.05$ ), lo que indica la conveniencia de extraer una (la única posible) función de discriminante, es decir, dicha función es significativa.

CUADRO 12. LAMBDA DE WILKS

\begin{tabular}{ccccc}
\hline Contraste de las funciones & Lambda de Wilks & Chi-cuadrado & gl & Sig. \\
\hline 1 &, 274 & 1270,389 & 10 &, 000 \\
\hline
\end{tabular}

Una vez confirmada la significatividad del eje discriminante, se calcula la correlación canónica la dicha función discriminante con la variable categórica que define los niveles de desempeño financiero, alto y bajo. El Cuadro 13, muestra la capacidad explicativa de la función discriminante obtenida. Una confirmación final de esta conclusión es que el porcentaje de varianza explicada por la función discriminante es del 100\%. El autovalor 2.646 (el cual es grande), indica que función discriminante discrimina mucho, ya que explica la mayor parte de la variabilidad total de la nube de puntos sobre la función discriminante.

\section{CuAdro 13. Autovalores}

\begin{tabular}{ccccc}
\hline Función & Autovalor & \% de varianza & \% acumulado & $\begin{array}{c}\text { Correlación } \\
\text { canónica }\end{array}$ \\
\hline 1 &, $274^{\mathrm{a}}$ & 1270,389 & 10 &, 000 \\
\hline
\end{tabular}

a. Se han empleado las 1 primeras funciones discriminantes canónicas en el análisis.

Ahora conviene conocer cuáles son las variables que tienen mayor poder discriminante en orden a clasificar a una empresa en uno de los dos grupos (alto desempeño financiero, Bajo desempeño financiero. Una forma de medir ese poder discriminante es calculando el coeficiente de correlación entre cada una de las variables (razones financieras) y la función discriminante. En el Cuadro 14 se muestra la matriz de estructura, en la cual se puede observar que la correlación de la función discriminante con la razón Circulante (0.852) es mayor en valor absoluto que con la razón Prueba de Ácido (0.710). Las comparaciones se deben de realizar siempre en valor absoluto. Estas variables aparecen ordenadas de acuerdo con el valor absoluto de los coeficientes de correlación en el cuadro siguiente. 


\section{CUADRO 14. MATRIZ DE ESTRUCTURA}

\begin{tabular}{|c|c|}
\hline & Función \\
\hline & 1 \\
\hline Circulante & 0.852 \\
\hline Prueba de ácido & 0.710 \\
\hline Capital de aportación ${ }^{\mathrm{a}}$ & 0.324 \\
\hline Capital de deuda ${ }^{a}$ & -0.323 \\
\hline Prueba rápida ${ }^{\mathrm{a}}$ & 0.293 \\
\hline Endeudamiento total ${ }^{\mathrm{a}}$ & -0.278 \\
\hline Dias de pagoa & -0.274 \\
\hline Rotación de inventarios ${ }^{\mathrm{a}}$ & -0.269 \\
\hline Días de cobro & 0.205 \\
\hline Margen de utilidad neta & 0.176 \\
\hline Rentabilidad del activoa & 0.130 \\
\hline RION después de impuestos ${ }^{\mathrm{a}}$ & 0.122 \\
\hline Gastos de operación ${ }^{\mathrm{a}}$ & -0.116 \\
\hline Rendimiento de la IONPRIONa & 0.107 \\
\hline Veces que se paga el interés ${ }^{\mathrm{a}}$ & 0.103 \\
\hline Rotación del activo & -0.092 \\
\hline Rotación del activo de largo plazo & 0.085 \\
\hline Rentabilidad del capital contable & 0.078 \\
\hline Interes a ventas ${ }^{a}$ & -0.275 \\
\hline Efectivo de operación interés & 0.066 \\
\hline Días de inventarios ${ }^{\mathrm{a}}$ & 0.065 \\
\hline Veces que se paga la pérdida cambiaria & 0.064 \\
\hline Apalancamiento & -0.052 \\
\hline Utilidad en operación ${ }^{\mathrm{a}}$ & 0.049 \\
\hline Pasivo $^{a}$ & 0.044 \\
\hline Interés a utilidad neta ${ }^{\mathrm{a}}$ & -0.042 \\
\hline Activo $^{a}$ & 0.037 \\
\hline Margen de utilidad bruta ${ }^{a}$ & -0.034 \\
\hline Activo de largo plazo ${ }^{a}$ & -0.030 \\
\hline Efectivo de operación a pérdida cambiaria & 0.026 \\
\hline Utilidad del ejercicioa & -0.024 \\
\hline Ventas $^{\mathrm{a}}$ & -0.022 \\
\hline Generación de efectivoa & -0.020 \\
\hline Tasa de provisiones ${ }^{\mathrm{a}}$ & 0.016 \\
\hline Capital contable ${ }^{\mathrm{a}}$ & 0.008 \\
\hline Grado de apalancamiento financiero ${ }^{a}$ & -0.005 \\
\hline Pasivo de largo plazo ${ }^{a}$ & 0.000 \\
\hline
\end{tabular}

Correlaciones intra-grupo combinadas entre las variables discriminantes y las funciones discriminantes canónicas tipificadas. Variables ordenadas por el tamaño de la correlación con la función

a. Esta variable no se emplea en el análisis. 
En el Cuadro 15 aparecen las puntuaciones de los centroides de las razones financieras con respecto a las funciones discriminantes. Con estos resultados, el punto de corte discriminante será el punto medio de las funciones en los centroides de los grupos:

Centroides de los grupos: $C=\frac{D_{1}+D_{2}}{2}=\frac{-1.385+1.907}{2}=0.261$

CuAdRo 15. Funciones DE LOS CENTROIDES DE LOS GRUPOS.

\begin{tabular}{lc}
\hline \multirow{2}{*}{ Desempeño financiero } & Función \\
\cline { 2 - 2 } & $\mathbf{1}$ \\
\hline Bajo desempeño financiero & $-1,385$ \\
\hline Alto desempeño financiero & 1,907 \\
\hline
\end{tabular}

Funciones discriminantes canónicas no tipificadas evaluadas en las medias de los grupos

\subsection{Estadísticos de clasificación}

Enseguida, se calcula el valor de las funciones de clasificación, y se clasifica a cada empresa en aquél grupo cuya función discriminante resulte tomar el mayor valor. De acuerdo con el Cuadro 16, las funciones de clasificación son:

Para el nivel de desempeño bajo:

$F_{1}=1.176 C+0.510 P A+26.213 A-0.030 V P P C+0.040 E O I+0.021 E O P C$

$+0.128 D C+23.925 R A+298.670 M U N-172.839 R C C-18.503$

Para el nivel de desempeño alto:

$\mathrm{F}_{1}=10.730 \mathrm{C}-2.503 P A+21.866 \mathrm{~A}-0.0150 \mathrm{VPPC}-0.060 \mathrm{EOI}+0.004 \mathrm{EOPC}$

$+0.148 D C+21.333 R A+246.0150 M U N-136.9259 R C C-27.451$

Para la aplicación de las funciones discriminantes, se calcula la puntuación de cada empresa en cada en cada uno de los grupos, utilizando las funciones clasificadoras. Finalmente, una empresa se clasifica en el grupo en el que ha alcanzado la puntuación más elevada. 


\section{CUADRO 16. COEFICIENTES DE LA FUNCIÓN DE CLASIFICACIÓN}

\begin{tabular}{lcc}
\hline & \multicolumn{2}{c}{ Desempeño financiero } \\
\hline Circulante & Bajo desempeño financiero & Alto desempeño financiero \\
\hline Prueba de ácido & 1.176 & 10.730 \\
\hline Apalancamiento & 0.510 & -2.503 \\
\hline $\begin{array}{l}\text { Veces que se paga la pérdida } \\
\text { cambiaria }\end{array}$ & 26.213 & 21.866 \\
\hline Efectivo de operación interés & -0.030 & -0.015 \\
\hline $\begin{array}{l}\text { Efectivo de operación a perdida } \\
\text { cambiaria }\end{array}$ & 0.040 & -0.060 \\
\hline Días de cobro & 0.021 & 0.004 \\
\hline Rotación del activo & 0.128 & 0.148 \\
\hline Margen de utilidad neta & 23.925 & 21.333 \\
\hline Rentabilidad del capital contable & 298.670 & 246.015 \\
\hline (Constante) & -172.839 & -139.925 \\
\hline
\end{tabular}

Funciones discriminantes lineales de Fisher

\section{CUADRO 17. RESULTADOS DE LA CLASIFICACIÓN $\mathrm{N}^{\mathrm{a}, \mathrm{c}}$.}

\begin{tabular}{|c|c|c|c|c|c|}
\hline & & \multirow{3}{*}{ Desempeño financiero } & \multirow{2}{*}{\multicolumn{2}{|c|}{$\begin{array}{l}\text { Grupo de pertenencia } \\
\text { pronosticado }\end{array}$}} & \\
\hline & & & & & \multirow{2}{*}{ Total } \\
\hline & & & $\begin{array}{l}\text { Bajo desempe- } \\
\text { ño financiero }\end{array}$ & $\begin{array}{l}\text { Alto desempe- } \\
\text { ño financiero }\end{array}$ & \\
\hline \multirow{4}{*}{ Original } & \multirow{2}{*}{ Recuento } & $\begin{array}{l}\text { Bajo desempeño } \\
\text { financiero }\end{array}$ & 568 & 5 & 573 \\
\hline & & $\begin{array}{l}\text { Alto desempeño finan- } \\
\text { ciero }\end{array}$ & 50 & 366 & 416 \\
\hline & \multirow{2}{*}{$\%$} & $\begin{array}{l}\text { Bajo desempeño } \\
\text { financiero }\end{array}$ & 99.1 & 0.9 & 100.0 \\
\hline & & $\begin{array}{l}\text { Alto desempeño finan- } \\
\text { ciero }\end{array}$ & 12.0 & 88.0 & 100.0 \\
\hline \multirow{4}{*}{$\begin{array}{l}\text { Validación } \\
\text { cruzada }^{b}\end{array}$} & \multirow{2}{*}{ Recuento } & $\begin{array}{l}\text { Bajo desempeño } \\
\text { financiero }\end{array}$ & 567 & 6 & 573 \\
\hline & & $\begin{array}{l}\text { Alto desempeño finan- } \\
\text { ciero }\end{array}$ & 52 & 364 & 416 \\
\hline & \multirow{2}{*}{$\%$} & $\begin{array}{l}\text { Bajo desempeño } \\
\text { financiero }\end{array}$ & 99.0 & 1.0 & 100.0 \\
\hline & & $\begin{array}{l}\text { Alto desempeño finan- } \\
\text { ciero }\end{array}$ & 12.5 & 87.5 & 100.0 \\
\hline
\end{tabular}

a. Clasificados correctamente el $94.4 \%$ de los casos agrupados originales.

b. La validación cruzada sólo se aplica a los casos del análisis. En la validación cruzada, cada caso se clasifica mediante las funciones derivadas a partir del resto de los casos

c. Clasificados correctamente el $\mathbf{9 4 . 1 \%}$ de los casos agrupados validados mediante validación cruzada 


\section{Discusión}

Al analizar las funciones discriminantes generadas en el Cuadro 7, se puede concluir que en la aplicación del análisis discriminante en la clasificación y predicción del desempeño financiero, las razones financieras: Circulante, Prueba de ácido, Apalancamiento, Veces que se paga la pérdida cambiaria, efectivo de operación interés, Efectivo de operación a pérdida cambiaria, Días de cobro, Rotación del activo, Margen de utilidad neta y Rentabilidad del capital contable presentan diferencias significativas en el nivel de desempeño financiero de las empresas del sector en estudio en el periodo comprendido entre el cuarto trimestre del año 2001 al tercer trimestre del año 2015. Por lo anterior, se puede inferir que los rubros asociados a estas razones financieras se ven afectados positivamente en el sector de productos de consumo frecuente que cotiza en la BMV.

Por otro lado, estudios desarrollados demuestran la efectividad de la metodología planteada por el Análisis Discriminante para la clasificación y predicción del nivel de liquidez, actividad, rentabilidad y endeudamiento de las empresas debido a la facilidad que este establece para el manejo de múltiples variables (Suárez, 2000).

Es importante mencionar que investigaciones desarrolladas han demostrado que los procesos de estandarización con diferentes normas inciden positivamente en el mejoramiento de indicadores de las organizaciones en diferentes sectores empresariales. Lo cual, también se demuestra en esta investigación (Fontalvo, Mendoza, \& Morelos, 2011a) y (Fontalvo, Morelos, \& De la Hoz, 2011b).

Es importante mencionar también, que en ésta investigación se presenta un método y un conjunto de herramientas que permiten clasificar y predecir el desempeño financiero de las empresas del sector de productos de consumo frecuente que cotizan en la BMV, es por esto que los autores (Vivanco, Martínez, \& Taddei, 2010) hacen uso de la metodología que proporciona el Análisis Discriminante para determinar los niveles de competitividad de varias empresas de acuerdo a ciertas variables específicas. Lo que demuestra la utilidad que tiene el ADM para la clasificación de empresas que presentan variables similares, pero las cuales es necesario diferenciarlas, además se presenta una explicación clara de las razones financieras seleccionadas que mejoran de un periodo a otro. 


\section{Conclusiones}

En este trabajo de investigación se utilizan 19 empresas del sector de productos de consumo frecuente que cotizan en la BMV, el modelo presenta una alta efectividad en la clasificación de los dos niveles estudiados. En la muestra original la precisión del modelo para el nivel de desempeño alto es $88.0 \%$, y para el nivel bajo es de $99.1 \%$, para un promedio total de clasificación del $94.4 \%$ de las empresas, lo que demuestra una excelente confiabilidad para predecir el comportamiento de las razones financieras en el sector a futuro.

De los resultados y la discusión se puede concluir que existen diferencias significativas en los indicadores de los dos niveles, como resultado de la aplicación del análisis discriminante en la clasificación del nivel de desempeño de las empresas del sector de productos de consumo frecuente que cotizan en la BMV. Sin embargo, la incidencia de estas razones financieras pueden cambiar en otro sector empresarial, como señalan investigaciones realizadas por algunos autores quienes afirman que la adopción de estándares puede afectar de manera positiva la competitividad y las razones financieras de la empresa o el sector que los implementa, ya que estos representan la estandarización de los procesos y la producción de productos sanos; analizando que tanto la competitividad como la gestión financiera están influenciadas positivamente por la adopción de estándares, ya que aquellas empresas que lo hicieron pudieron mantenerse en el mercado y aumentar su participación (Avendaño \& Varela, 2010).

De igual forma, esta investigación permite establecer una función objetivo para las empresas analizadas en el sector de productos de consumo frecuente que cotizan en la BMV, con lo que se puede estudiar y analizar las razones financieras que discriminan mejor y así poder tomar acciones teniendo en cuenta el cálculo y estudio de las razones financieras de liquidez, actividad, rentabilidad y endeudamiento que presentaron una buena discriminación.

Finalmente, se puede comprobar que del modelo propuesto en ésta investigación, se puede alcanzar el objetivo del análisis discriminante como son: primero examinar las diferencias entre los niveles estudiados, es decir, los niveles de desempeño financiero, al to y bajo, en cuanto a su comportamiento con respecto a las variables consideradas. Y como segundo objetivo, se puede realizar una clasificación sistemática de las razones financieras seleccionadas.

A partir de estudios como éste, se puede realizar análisis en otros sectores empresariales que faciliten la toma de decisiones sobre la determinación de 
las variables, rubros y razones financieras que redundan en el mejoramiento de la situación productiva de las organizaciones y poder realizar otras razones financieras que incidan en el posicionamiento de otros sectores estudiados. Se recomienda para futuros estudios incrementar el número de razones financieras e incorporar indicadores de competitividad; y se invita a analizar el comportamiento de otros sectores empresariales por medio de esta metodología.

\section{Bibliografía}

Altman, E. (1968). Financial ratios, discriminant analysis, and the prediction of corporate bankruptcy. Journal of Finance, 589-609.

Altman, E., Hadelman, R., \& Narayanan, P. (1977). Zeta analysis, a new model to identify bankruptcy of corporations. Journal of Banking and Finance, 29-51.

Avendaño, B., \& Varela, R. (2010). La Adopción de estándares en el sector hortícola de baja california. Estudios Fronterizos, 11(1), 171-202.

Beaver, W. (1966). Financial ratios as predictors of failure. Journal of Accounting Research, 5(Suppl), 71-102.

Brealey, R., \& Myers, S. (1998). Principios de finanzas corporativas. España: Mc Graw-Hill.

De la Garza, J., Morales, B., \& González, B. (2013). Análisis estadístico multivariante. Un enfoque teórico y práctico. México D.F.: McCraw-Hill.

Díaz, N. (2009). Ranking financiero: Herramienta financiera para medir la productividad de las empresas de comercialización de energía eléctrica. 27(111), 13-34.

Fizpatrick, P. (1931). Symptoms of Industrial Failure. Washington D.C.: Catholic University of American Press.

Fontalvo, T., Mendoza, A., \& Morelos, ]. (2011a). Evaluación del impacto de los sistemas de gestión de la calidad en la liquidez y rentabilidad de las empresas de la Zona Industrial de Mamonal (Cartagena-Colombia). Revista Católica del Norte, 1(1), 1-28.

Fontalvo, T., Morelos, J., \& De la Hoz, E. (2011b). Aplicación del análisis discriminante para evaluar el mejoramiento de los indicadores financieros en las empresas del Sector extracción de petróleo crudo y gas natural en Colombia. Revista Soluciones de Postgrado EIA, 1(1), 1-16.

Fontalvo, T., Vergara, J., \& De la Hoz, E. (2012). Evaluación del impacto de los sistemas de gestión de la calidad en la liquidez y rentabilidad de las empresas de la Zona Industrial. Pensamiento \& gestión, 1(1), 3-25. 
Gil, J., García, E., \& Rodríguez, G. (2001). Análisis discriminante. Madrid: La Muralla S.A. Guajardo, G. (2002). Contabilidad financiera. México: Mc Graw-Hill.

Ibarra, A. (2010). Desarrollo del Análisis Factorial Multivariable Aplicado al Financiero Actual. Edición electrónica gratuita. Texto completo. Recuperado el 06 de 05 de 2017, de http://www.eumed.net/libros-gratis/2010a/666/indice.htm

Lachenbruch, P. (1975). Discriminant análysis. 47.

Levy, L. (2004). Planeación financiera en la empresa moderna: el mejoramiento estratégico de las finanzas para lograr el éxito empresarial. México ISEF, 20(4), 35-50.

Nava, R., \& Marbelis, A. (2009). Análisis financiero: una herramienta clave para una gestión financiera eficiente. Revista Venezolana de gerencia, 14(48), 606-628.

Nuñez, A., \& Vieites, R. (2009). El diagnóstico financiero de la empresa. Ceei Calicia S.A., 12(2), 5-32.

Ortíz, H. (2011). Análisis financiero aplicado.

Palacio, J. (2008). Importancia del diagnóstico económico financiero para conocer su posición económica financiera de la empresa. Altair Consultores, 14(2), 5-20.

Perez, C. (2004). Técnicas de análisis multivariante de datos. Madrid: Pearson Educación S.A.

Russ, R., Achilles, W., \& Greenfield, J. (2009). The Altman Z-Score Revisted. Journal of Internactional Finance $\&$ Economics, 9(4), 59-73.

Somoza, A. (2000). Los modelos contables-financieros de predicción de la insolvencia empresarial. Una aportación y su aplicación a una muestra de empresas de los sectores textil y confección de la provincia de Barcelona (1994-1997). (U. d. Comptabilitat, Ed.) Recuperado el 08 de 05 de 2017, de Tesis Doctorales en Red: http://www. tesisenred.net/handle/10803/1254

Suárez, ]. (2000). Los parámetros caracterisitcos de las empresas manufactureras de alta rentabilidad: una aplicación del análisis discriminante. Revista española de financiación y contabilidad, 29(1), 443-481.

Tabachnick, B., \& Fidell, L. (2001). Using multivariate estatistics (Sixth ed.). EE.UU.: Pearson Education, Inc.

Tricova, M., \& Terdovski, D. (2008). Applie discriminant análisis in estimation of potential EU members. Businesstatistics-economic informatics, June, 32(120), 48.

Vázquez, X., Guerra, A., \& Ahmed, I. (Octubre de 2008). Aplicación de métodos multivariados: una respuesta a las limitaciones de los ratios financieros. Revista de la Universidad de Granma, 24(114), 44.

Vivanco, M., Martínez, F., \& Taddei, I. (2010). Análisis de competitividad de cuatro sistema-producto estatales de tilapia en México. Estudios Sociales, 18(1), 165-207. 
Vuran, B. (2009). Prediction of business failure: a comparison of discriminant and logistics regression analyses. Istanbul University journal of the school of business administration, September, 38(1), 47-65. 